\title{
http://dx.doi.org/10.21611/qirt.1994.044 \\ Photothermal detection of surface defects and thermal changes in near-surface layers
}

\author{
by SCHMITZ B., REICK M., GOCH G. * and STEINER R.
}

Institut für Lasertechnologien in der Medizin an der Universität UIm (ILM), Arbeitsgruppe Lasermeßtechnik, Helmholtzstr. 12, D-89081 UIm, FRG

"Universität Ulm, Abteilung Meß-, Regel- und Mikrotechnik (MRM), Bereich Meßtechnik, Albert-Einstein-Allee 41, D-89069 UIm, FRG

\begin{abstract}
When mechanical or structural changes on surfaces and in near-surface layers are correlated with alterations of thermal properties, they become detectable by means of photothermal microscopy. We applied this approach to the detection of variations in ceramic materials $\left(\mathrm{Si}_{3} \mathrm{~N}_{4}\right.$, Widianit $\left.\mathrm{N2000}\right)$ induced by Vickers indentations of several loads. The photothermal images show remarkable changes near the marked areas depending on the different loading strengths. Certainly cracks, but probably also alterations of the microcrystalline structure are responsible for this behavior. This statement is confirmed by thermal diffusivity measurements and it agrees with estimations known from the photothermal defect identification.
\end{abstract}

\section{Introduction}

From conventional machining and precision engineering it is well known that various mechanical, physical, chemical or thermal impacts generate the state of the surface and nearsurface layers. Each individual impact, which may occur in a continuous or intermittent mode, has a certain influence on the material's physical properties. For example, mechanical processing steps result in strain hardening, plastic deformation or compressive residual stress - thus, affecting the geometry and the accuracy of a machined part. Thermal impacts may lead to surface annealing, tensile workpiece stresses or phase transitions. Since all these properties determine decisively the functional behavior under working conditions, it is of great importance to know the state of the surface and near-surface layers - in particular both the mechanically or thermally induced inhomogeneities and the variations of the physical, thermal and mechanical properties. But, most of the workpiece properties and their changes due to machining can neither be estimated nor calculated. They have to be measured in a nondestructive, contactless manner and with a high spatial and depth resolution [1].

The depth of the variations ranges from several $\mathrm{mm}$ up to about $1 \mathrm{~mm}$. Owing to the main advantages of the photothermal principle that it is suitable for near-surface inspection and that the measuring depth can be adjusted by different modulation frequencies, a change of thermal properties becomes detectable by means of photothermal microscopy. The surface properties, the variations and depth profiles of the thermal parameters can be distinguished from the features of the base materials.

Our special interest in this field is focused on the detection of altered zones caused by a manufacturing process and the thermal variations in near-surface layers due to mechanical or

The colour plates of this article are on page XVI at the end of the book. 
http://dx.doi.org/10.21611/qirt.1994.044

laser material processing. Basically, we assume that the varied mechanical properties, caused by locally distributed inhomogeneities on and beneath the sample's surface (e.g. cracks, delaminations, alterations of microcrystalliné structures, varied material densifications, porosities, etc.) are correlated to changes of the thermal properties. In fact, while scanning the sample with a heating beam, we observe an image blurring due to subsurface structures affecting the penetration of the thermal waves [2]. In order to clarify this problem we determine the thermal properties, especially the thermal diffusivities and we examine the basic conditions for a reliable identification of thermal inhomogeneities and subsurface structures.

In the following, we present experimental results on ceramic materials. The theoretical survey will be very compressed; a more detailed study is given in $[3,4]$. The experimental configuration has been explained elsewhere in detail [2]. In order to model the changes of material properties by a slight mechanical load we chose the Vickers hardness check.

\section{Survey of the theoretical basis for the identification of subsurface thermal inhomogeneities}

Recently, in analogy to the classical optical microscopy Walther et. al. [3,4] proposed a complex valued photothermal point spread function (PSF) $G\left(x, y, z_{0}\right)$, which describes the signal distribution of a buried point-like defect while scanning the sample's surface with a modulated heating laser beam. Comparing the photothermal signal influenced by the defect $S_{0}+S_{1}$ (which consists of a contribution due to the defect $S_{1}$ and the background signal $S_{0}$ ) with the background signal $S_{0}$ of the surrounding homogeneous material, a contrast function - both for the amplitude and phase signals - can be defined. The contrasts are reflected by the real and the imaginary parts of the photothermal PSF convoluted with the defect distribution $O\left(x, y, z_{0}\right)$.

$$
\begin{array}{lll}
\text { Amplitude contrast : } & \mathrm{K}_{\mathrm{A}}=\frac{\operatorname{Amp}\left(\mathrm{S}_{0}+\mathrm{S}_{1}\right)-\operatorname{Amp}\left(\mathrm{S}_{0}\right)}{\operatorname{Amp}\left(\mathrm{S}_{0}\right)}=\mathfrak{R e}\left(\mathrm{G}^{*} \mathrm{O}\right) \\
\text { Phase contrast: } & \mathrm{K}_{\varphi}=\varphi_{\mathrm{s}_{0}+\mathrm{s}_{1}}-\varphi_{\mathrm{s}_{0}}=\operatorname{Im}\left(\mathrm{G}^{*} \mathrm{O}\right)
\end{array}
$$

Walther et. al. found out that the photothermal contrasts depend on the normalized defects' depths $z_{0} / m_{\text {th }}$ (which is proportional to the square root of the modulation frequency $f^{1 / 2}$ ) and the distortions of the thermal parameters $k$ (thermal conductivity) and rc (heat capacity per unit volume). The dependence results in extrema and zero-crossings due to the modified interference conditions of the thermal waves. Obviously, in order to achieve a good contrast several measurements have to be performed at several modulation frequencies close to the extremas - both amplitude and phase. Furthermore, the photothermal resolution power depends on the modulation frequency and the defects' characteristics. Consequently, the modulation frequency is a decisive parameter for achieving best distinction of subsurface defects. Those mainly theoretical results lead to experimental conditions expecting the best photothermal distinction of any variation of thermal properties on the surface or in near-surface layers of a machined part.

\section{Experimental results - investigation of ceramic materials}

With respect to their further applicability, reliability and functionality advanced ceramics have to fulfill high demands on shape, accuracy and surface finishing. After the sintering process the ceramic workpieces have to be ground in rough machining and, finally, finished with diamond wheels. Due to the mechanical and thermal load brittle fracture, surface defects, 
http://dx.doi.org/10.21611/qirt.1994.044

cracks and subsurface damage may be induced by the material removal and increased material compressions. Plastic deformations are caused by the impacts of local pressures and heat in the contact zones during the machining process [5]. Since these processes have a significant influence on the mechanical strength and the later use, their effects on materials' properties have to be determined - non-destructively and contactless. Again, as these changes happen in near-surface layers and are probably combined with variations of the thermal properties, they become detectable by photothermal means [6].

In order to induce a mechanical impact (comparable with a mechanical load during machining) onto a solid material, we investigated experimentally ceramic (SiliconNitride $\mathrm{Si}_{3} \mathrm{~N}_{4}$ WIDIANIT 2000) and hard metal (Tungsten Carbide and Titan Carbide/Nitride with different amounts of Cobalt and Nickel - WC-Co TTI25, Cermet THM) samples, where a Vickers indenter with various pressures ( $2 \mathrm{~kg}-50 \mathrm{~kg}, \mathrm{HV} 2$ - HV50) was pressed into. Color figures 1 and 2 illustrate for example only two of the obtained results. A Vickers tip of 163 degrees was pressed into a ceramic sample of $\mathrm{Si}_{3} \mathrm{~N}_{4}$ (WIDIANIT 2000). Before the test the sample had been polished. The marked areas were scanned using both the optical beam deflection and the radiometric detection methods. Here, the figures display two of the radiometrically obtained results.

Obviously, the photothermal images show a remarkable signal variation at the areas surrounding the Vickers distorted surface, comprising both the amplitude and the phase image. Figure 1 shows the considerable increase of the amplitude signal where an impact of 2 $\mathrm{kg}$ (HV2) affected the surface. The intender deformed an area of about $35 * 35 \mathrm{~mm}^{2}$ and penetrated less than $4 \mathrm{~mm}$ into the material. An area of 200 * $160 \mathrm{~mm}^{2}$ was scanned and photothermally registered. The phase image shown in figure 2 corresponds to this result. Here, the Vickers indenter was weighted with $10 \mathrm{~kg}$ (HV10) deforming an area of $75 * 75 \mathrm{~mm}^{2}$ and penetrating about $8 \mathrm{~mm}$ into the sample's surface. Cracks starting at the rectangular corners (also traceable in the optical microscopic images) were clearly resolved. Near the edges the phase angle decreases about 2 degrees. Opposite to these thermal images no structure in the optical images could be resolved which is comparable to the thermally obtained features except the Vickers indented surface and the cracks. Furthermore, the increased amplitude and phase signals in the neighborhood of the Vickers marked area could not be seen optically.

These results indicate that due to the mechanical load the thermal flux into the sample is disturbed. The reasons for this behavior are not yet completely understood. Probably, there are parallel subsurface median cracks below the sample's surface. Or, (it is a well known fact) the changes of the thermal properties are correlated to modifications of the microcrystalline structure. Both effects might be caused by the mechanical load. The cracks starting near the rectangular corners (see figure 2) support the first explanation, while the wide areas of varied signal magnitudes near the edges refer to the second one.

In order to clarify this question we measured the amplitude and phase on varied loaded areas near and far from the Vickers indented surfaces as a function of the modulation frequency. The contrasts are shown in figure 3.

These figures demonstrate that there is a clear indication that both the amplitude and the phase contrasts carry information about the outer structure and the varying thermal properties near the surface. Both signals depend on the different loading strengths. Considering only the two HV30 curves we want to emphasize the very good agreement between the theoretical calculation based on the photothermal point spread function and the experimental results. 
http://dx.doi.org/10.21611/qirt.1994.044
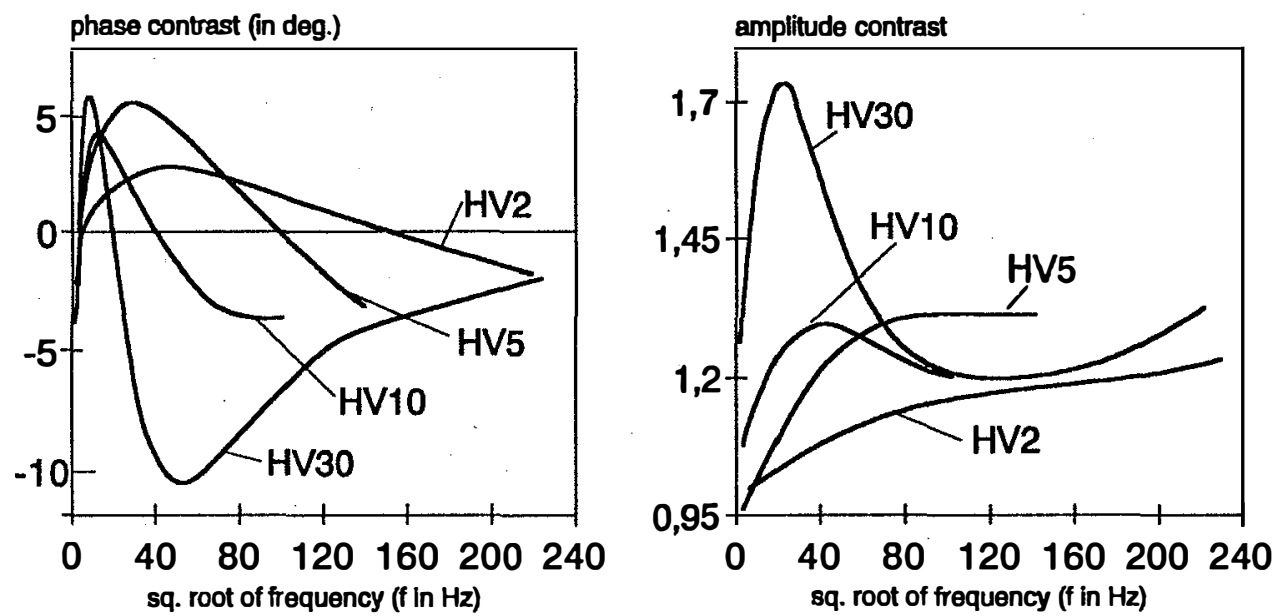

Fig. 3: Phase (left) and amplitude (right) contrasts as functions of the modulation frequency for different loaded Vickers indented areas.

Assuming that the absorption coefficient of the Vickers marked area increased to about 1.3 times of the surrounding value, the position of the maximum amplitude is near to the zerocrossing of the phase contrast and, vice versa, the minimum phase is near to the 'first zerocrossing' of the amplitude contrast function. Unfortunately, a second zero-crossing could not be observed due to the decreasing signal in the high frequency range. Furthermore, in order to prove our explanations we also determined the change of the thermal properties far and near the marked areas. A considerable change of the thermal diffusivities, determined by the 'ZeroCrossing' method of Kuo et al. [7] was measured. The significantly, partly increasing, partly decreasing values certainly indicate that different reasons - subsurface cracks and changes of the microcristalline structures yield those thermal effects.

Table 1: Change of the thermal diffusivities caused by a mechanical load

\begin{tabular}{|l|l|l|l|l|}
\hline Material & $\mathrm{Si}_{3} \mathrm{~N}_{4}$ & WIDIANIT & TTI 25 & THM \\
\hline Thermal conductivity (in W/m $\mathrm{K}$ ) (1) & 35 & 29 & 15.7 & 80 \\
\hline Density (in g/cm3) (1) & 3.3 & 3.26 & 7 & 14.9 \\
\hline Hardness HV30 (1) & 1800 & 1400 & 1450 & 1580 \\
\hline Hardness HV50 (2) & - & - & 1480 & 1670 \\
\hline T.D. near (in cm $\mathrm{cm}^{2}$ ) (2) & $0.136-0.155$ & $0.178-0.216$ & $0.133-0.138$ & 0.258 \\
\hline T.D.outside (in cm $/ \mathrm{s}$ ) (2) & 0.147 & 0.199 & 0.105 & 0.261 \\
\hline
\end{tabular}

1: manufacturer; 2: this work; T.D. outside: thermal diffusivity, no influence of the marked area; T.D. near: thermal diffusivity, near and influenced by the marked areas, minimal and maximal values

Altogether, these figures and results justify the assumption that the physical properties of the ceramic samples have been changed in the surroundings of the Vickers deformed areas. The mechanical impacts yield changes of thermal properties and, hence, become detectable by photothermal techniques. 


\section{Conclusions}

It was the purpose of this contribution to demonstrate that photothermal measurement techniques can be applied for the detection of changes of mechanical and thermal properties due to a mechanical impact onto a solid sample. The verification of the fundamental assumption that mechanical changes are combined with variations of the thermal properties had been illustrated on ceramic materials. But, despite of these results it is still one of the main problems to include theoretical aspects into convenient experimental instruments and verified results. A three dimensional heat flux inside the thermally inhomogeneous sample must be considered. Further on, algorithms for image reconstruction and inversion techniques are necessary for an improved and reliable material characterization.

But nevertheless, the results of section 3 demonstrate that the photothermal techniques can be used to examine the changes of material properties in near-surface layers caused by an individual mechanical impact. Further work will be done to verify and improve these results. The experiments will be extended to other mechanically and thermally processed samples. This might finally yield a sensitive and reliable control of manufacturing processes.

\section{Acknowledgments}

The authors thank Jürgen Geerkens and Michael Reigl for their help in the theoretical calculations; Dr. K. Dreyer / Krupp Widia, Essen for the preparation of the ceramic samples and Dr. Hans R. Schubach / Zwick Materialprüfung, Ulm, who performed the Vickers indentations. The fruitful and brainstorming discussions with Uwe Seidel and Heinz G.Walther from the Friedrich-Schiller-Universität Jena are gratefully acknowledged. This work is partly supported by a grant of the federal state of Baden-Württemberg, Germany Nr. II 7532.292-4/1.

\section{REFERENCES}

[1] BRINKSMEIER (E.): State-of-the-art of non-destructive measurement of sub-surface material properties and damage, Precision Engineering 11, 4, (1989), 211-2,24

[2] REIGL (M.), GAPP (M.),SCHMITZ (B.), STEIN (J.), GOCH (G.), SEIDEL (H.) WALTHER (G.): Investigations of subsurface structures and buried inhomogeneities by photothermal inspection, Quantitative Infrared Thermography QIRT'94, Sorrento 23.-26.8.1994

[3] FRIEDRICH (K.), HAUPT (K.), SEIDEL (U.), WALTHER (H.G.): Definition, resolution and contrast in photothermal imaging, J.Appl.Phys. 72, 8, (1992), 3759-3764

[4] SEIDEL (U.), HAUPT (K.), WALTHER (H.G.): Analysis of the detectability of buried inhomogeneities by means of photothermal microscopy, J.Appl.Phys. 75, 9, (1994), 4396-4401 [5] TÖNSHOFF (H.K.), TRUMPOLD (H.), BRINKMEIER (E.), WOBKER (H.G.): Evaluation of Surface Layers of Machined Ceramics, Annals of the CIRP 38, 2, (1989), 699-708

[6] GOCH (G.), GEERKENS (J.), REICK (M.), SCHMITZ (B.): Analysis of surface layer variations by photothermal means, 8th International Topical Meeting on Photoacoustic and Photothermal Phenomena, Guadeloupe, France, to be published in Journal de Physique (1994)

[7] KUO (P.K.), LIN (M.J.), REYES (C.B.), FAVRO (L.D.), THOMAS (R.L.), KIM (D.S.), ZHANG (S.Y.), INGLEHART (L.J.), FOURNIER (D.), BOCCARA (A.C.), YACOUBI (N.): Mirage-Effect Measurement of Thermal Diffusivity. Part I: Experiment, Can. J. Phys. 64, (1986), 1165-1167 KUO (P.K.), SENDLER (E.D.), FAVRO (L.D.), THOMAS (R.L): Mirage-Effect Measurement of Thermal Diffusivity. Part II: Theory, Can. J. Phys. 64, (1986), 1168-1171 